\title{
Ectopic Hepatocellular Carcinoma Arising in the Left Triangular Ligament of the Liver
}

\author{
Ryu Kanzaki Terumasa Yamada Kunihito Gotoh \\ Hidenori Takahashi Hiroaki Ohigashi Osamu Ishikawa \\ Department of Surgery, Osaka Medical Center for Cancer and Cardiovascular \\ Diseases, Osaka, Japan
}

\section{Key Words}

Hepatocellular carcinoma - Ectopic · Left triangular ligament

\begin{abstract}
Hepatocellular carcinoma (HCC) rarely originates in extrahepatic liver tissue. Laparoscopic resection is widely used to treat HCC. This report presents a case of a patient with ectopic HCC arising in the left triangular ligament of the liver that was successfully treated by laparoscopic resection. A 59-year-old female presented with an elevated serum alpha-fetoprotein (AFP) level $(2,508 \mathrm{ng} / \mathrm{ml})$. Dynamic computed tomography demonstrated a tumor measuring $20 \mathrm{~mm}$ in diameter below the left diaphragm just adjacent to the spleen. The tumor showed contrast enhancement in the hepatic arterial phase and became less dense than the liver parenchyma in the portal phase. The patient was diagnosed with ectopic HCC arising in the left diaphragm and laparoscopic surgery was performed. The tumor was located in the left triangular ligament of the liver and had a stalk between the tumor and the liver. The tumor was resected, and the final diagnosis was moderately differentiated ectopic HCC arising in the left triangular ligament of the liver. The patient had an uneventful postoperative recovery and has experienced no recurrence over 18 months after the operation.
\end{abstract}

\section{Introduction}

Extrahepatic liver tissue can arise in various locations such as the gallbladder, intraabdominal ligaments, omentum, retroperitoneum and thorax. The incidence of extrahepatic liver tissue is $0.24-0.47 \%[1,2]$. Hepatocellular carcinoma (HCC) arising from extrahepatic liver tissue is even more rare; only 46 cases have been reported including the present case. This report presents the case of a patient with ectopic HCC arising in the left triangular ligament of the liver that was successfully treated by laparoscopic resection. 


\section{Case Report}

A 59-year-old female presented with an elevated serum alpha-fetoprotein (AFP) level (42 ng/ml) detected in a routine medical checkup. Her medical history revealed that she had undergone a hemithyroidectomy for papillary carcinoma of the thyroid. Computed tomography (CT) and abdominal ultrasonography taken at presentation showed no remarkable findings. She regularly received medical checkups. The serum AFP level rose up to $2,508 \mathrm{ng} / \mathrm{ml}$ over a two-year follow-up period. She was referred to the hospital for further examination. She had no history of alcohol abuse, and her family history was noncontributory. On admission, her abdomen was soft, without palpable masses. Complete blood counts, serum electrolytes and biochemical markers were within normal limits. The level of prothrombin induced by vitamin $\mathrm{K}$ absence or antagonist II (PIVKA-II), carcinoembryonic antigen, carbohydrate antigen (CA) 19-9, and CA 125 were within normal limits. Hepatitis B surface antigen and hepatitis $C$ virus antibody were negative. The indocyanine green retention rate at $15 \mathrm{~min}$ (ICG-R15) and coagulation test results were within normal ranges.

Dynamic CT demonstrated a round tumor measuring $20 \mathrm{~mm}$ in diameter below the left diaphragm just adjacent to the spleen (fig. 1). The tumor showed contrast enhancement in the hepatic arterial phase and became less dense than the liver parenchyma in the portal phase. An angiographic examination revealed the tumor to be fed by the dorsolateral branch of the hepatic artery (A2). CT during hepatic arteriography and CT during arterioportography showed a pattern of early enhancement and washout.

Three 5- to $12-\mathrm{mm}$ trocars were inserted under general anesthesia. The liver had a normal appearance. The tumor was located in the left triangular ligament of the liver and had a stalk between the tumor and the liver (fig. $2 a$ ). No invasion to the diaphragm or spleen was observed. The tumor was examined using a prototype laparoscopic near infrared camera system (Hamamatsu Photonics K.K., Hamamatsu, Japan), and the tumor showed bright fluorescent signals (fig. 2b). The tumor was resected with an adequate margin using electrocautery and ultrasonic shears. Macroscopically, the resected tumor was encapsulated, $20 \mathrm{~mm}$ in diameter, and light tan in color. Microscopically, the mass was a medullary and trabecular carcinoma mixed with occasional acinar structures.

The final diagnosis was moderately differentiated ectopic HCC arising in the left triangular ligament of the liver (fig. 3 ). Part of the mass was normal liver tissue containing portal triads. The pedicle connecting the ectopic liver to the liver contained blood vessels. The patient had an uneventful postoperative recovery and was given no postoperative therapy. To date, at 18 months after the operation, she has experienced no recurrence.

\section{Discussion}

Ectopic HCC is a rare clinical entity which is defined as HCC arising from extrahepatic liver tissue. Collan et al. classified extrahepatic liver tissue into four types: accessory liver lobe, small accessory liver lobe, ectopic liver, and microscopic ectopic liver [3]. An accessory liver lobe is a large liver lobe attached to the mother liver by a stalk. A small accessory liver lobe is a liver lobe weighing 10-40 g, attached to the mother liver. Ectopic liver is liver tissue which is not connected to the mother liver and usually is attached to the gallbladder or intraabdominal ligaments. Microscopic ectopic liver is a small mass or liver tissue found occasionally on the wall of the gallbladder.

Extrahepatic liver tissue can occur in various locations such as the gallbladder, hepatic ligaments, omentum, retroperitoneum and thorax. The incidence of extrahepatic liver tissue is $0.24-0.47 \%[1,2]$. Benign or malignant lesions can arise from extrahepatic liver tissue. Benign lesions are less frequent and about ten cases including pedunculated hemangiomas, adenomas and focal nodular hyperplasia have been reported. 46 cases of ectopic HCC were identified including the current case. The patients included 34 males and 12 females with a mean age of 62 years (range 34-81 years). 40 were Asian patients, mostly Japanese. The common characteristics of the majority of these patients, including the present, are that the mother livers were not cirrhotic, that there was neither HBV nor $\mathrm{HCV}$ infection and that serum AFP levels were high. 
Liver cirrhosis is a precancerous state, and more than $80 \%$ of patients in the general population who develop HCC have cirrhosis. In contrast with the general population, cirrhosis of the liver itself was identified in only 8 of the 46 cases with ectopic HCC. Although serologic testing for HBV or HCV is not available for all reported cases, only two patients demonstrated positive HBV marker and five patients demonstrated positive HCV marker. Few of the lesions, including the one observed in the present case, showed normal liver parenchyma around the HCC. The normal liver parenchyma must have been completely replaced by the HCC. Furthermore, the serum AFP levels were higher than $1,000 \mathrm{ng} / \mathrm{ml}$ in 20 of 46 patients (43\%), which, according to a Japanese survey, is higher than that in HCC (28\%) [4]. Based on these findings, HCC could arise in extrahepatic liver tissue before or in the absence of cirrhosis, and it is likely that nonviral factors are involved in the carcinogenesis. The present case had the common characteristics of ectopic HCC described above, i.e., the mother livers were not cirrhotic, there was neither HBV nor HCV infection, and serum AFP levels were high.

Extrahepatic liver tissue could have increased neoplastic potential because the abnormal arterial supply or venous and biliary drainage within the extrahepatic liver tissue may increase the tissue's exposure to various chemical carcinogens $[5,6]$. The ectopic HCC was located in the left triangular ligament of the liver in 4 of the 46 patients. Those located in the left triangular ligament, excluding the current case, were revealed because of intraperitonial bleeding due to rupture of the tumor. Rupture of the tumor was observed in 5 of 46 patients. A tumor in the left triangular ligament of the liver may be prone to rupture because of mechanical force generated by movement of the diaphragm.

Ectopic HCCs are surgically resected if possible, with a favorable prognosis. The resection of an ectopic HCC can be curative because the carcinogenetic potential of these tumors is usually limited to the ectopic liver itself [5]. Laparoscopic resection of HCC is a safe and feasible alternative to open surgery if the indications are appropriate [7]. As a result, two cases were thus identified, including the present case, of laparoscopically resected ectopic HCC [8].

A novel ICG fluorescence imaging technique can be used to visualize HCC [9]. ICG is intravenously injected at a dose of $0.5 \mathrm{mg} / \mathrm{kg}$ body weight several days before surgery. HCC nodules show bright fluorescent signals that can be visualized during surgery and completely removed with negative margins under the guidance of a prototype laparoscopic near infrared camera system (Hamamatsu Photonics K.K., Hamamatsu, Japan), which activates ICG with emitted light at a wavelength of $760 \mathrm{~nm}$ and filters out light with a wavelength below $820 \mathrm{~nm}[10,11]$. The ectopic HCC in the present case showed bright fluorescent signals, which were easily detected by PDE. Although the mechanisms that mediate the accumulation of ICG in HCC nodules remain unclear, it is thought that ICG accumulates in regions of tissue possessing leaky capillaries or of the disruption of bile secretion [9].

In conclusion, laparoscopic resection is a safe and minimally invasive surgical method to remove an ectopic HCC arising in the left triangular ligament of the liver. 
Fig. 1. Dynamic CT image. a Transectional view. b Sagittal view. Dynamic CT demonstrated a round tumor measuring $20 \mathrm{~mm}$ in diameter below the left diaphragm just adjacent to the spleen (arrow).

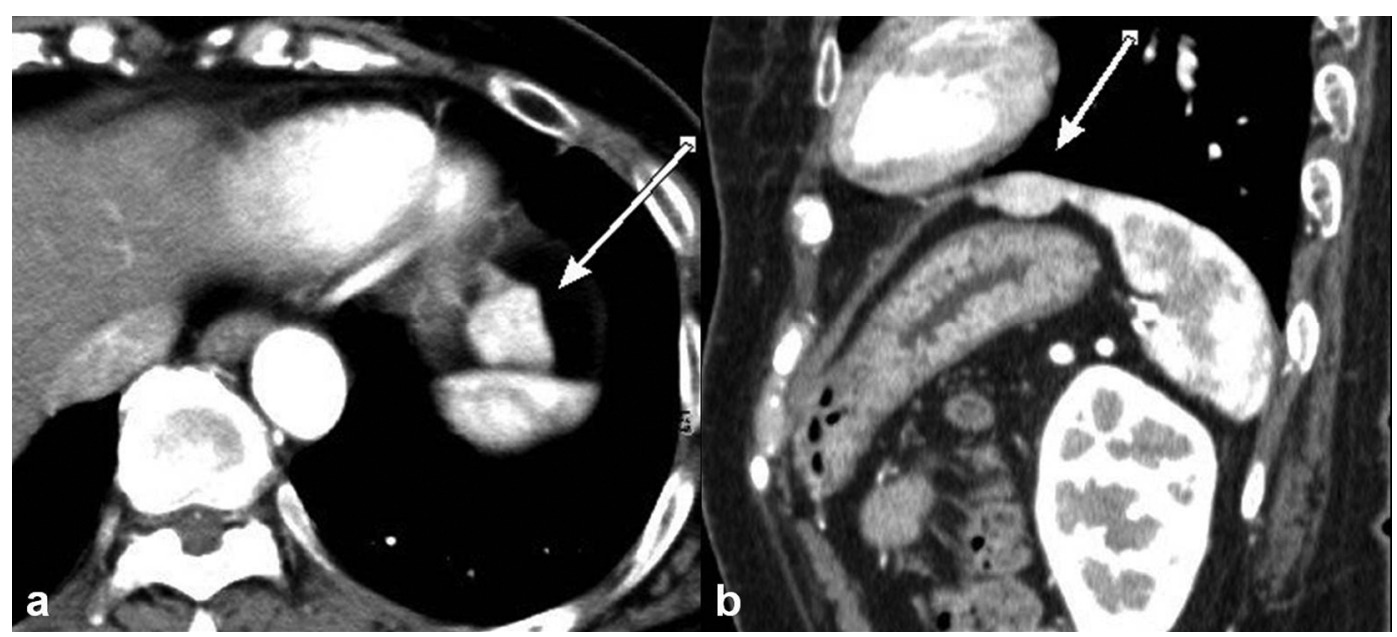

Fig. 2. Surgical view. a Laparoscopic view. The tumor was located in the left triangular ligament of the liver and had a stalk between the tumor and the liver. $\mathbf{b}$ The tumor was examined using a near infrared camera system (PDE; Hamamatsu Photonics K.K., Hamamatsu, Japan). The tumor showed bright fluorescent signals.

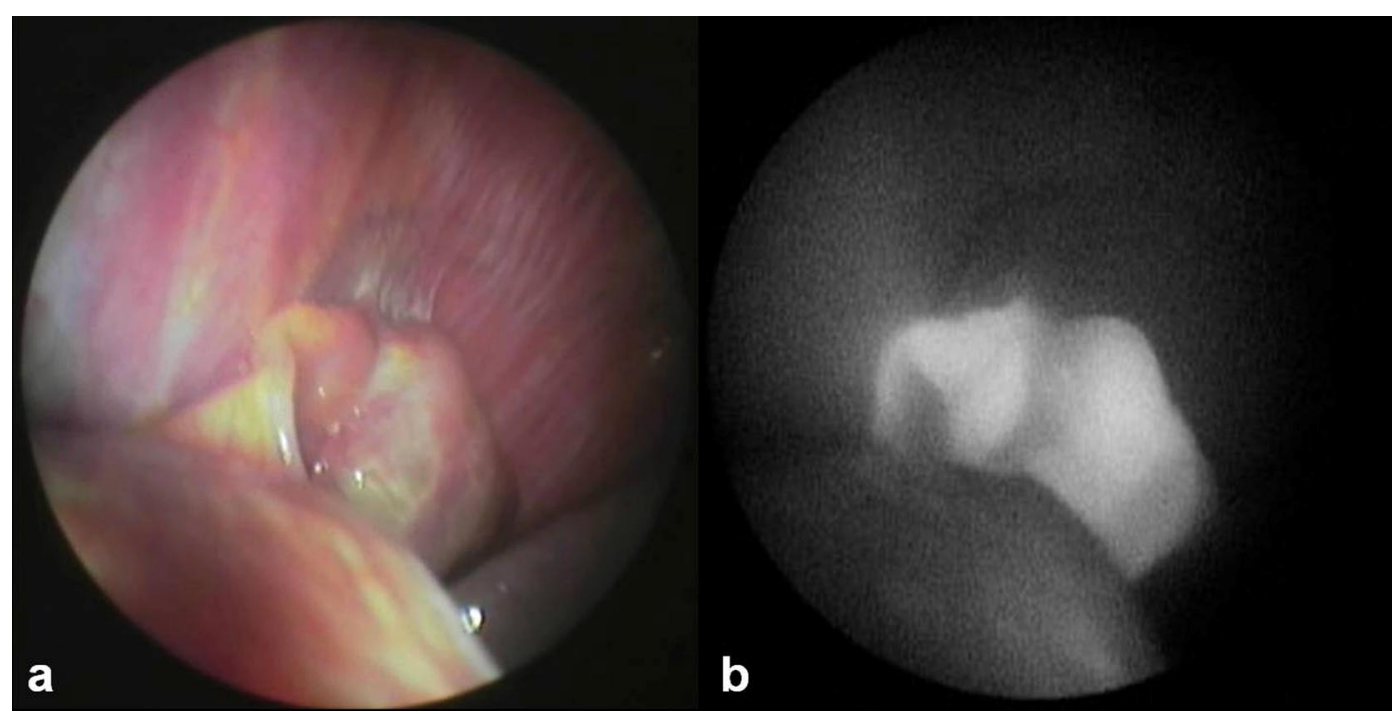




\begin{tabular}{r|l|l|l}
$\begin{array}{r}\text { Case Reports in } \\
\text { Gastroenterology }\end{array}$ & $\begin{array}{l}\text { Case Rep Gastroenterol 2010;4:138-143 } \\
\text { D0I: 10.1159/000314042 }\end{array}$ & Published online: May 12, 2010 & $\begin{array}{l}\text { O 2010 S. Karger AG, Basel } \\
\text { ISSN 1662-0631 } \\
\text { www.karger.com/crg }\end{array}$ \\
\hline
\end{tabular}

Fig. 3. Microscopic examination of the tumor. HE stain, $\times 100$. Microscopic examination of the tumor confirmed moderately differentiated HCC.

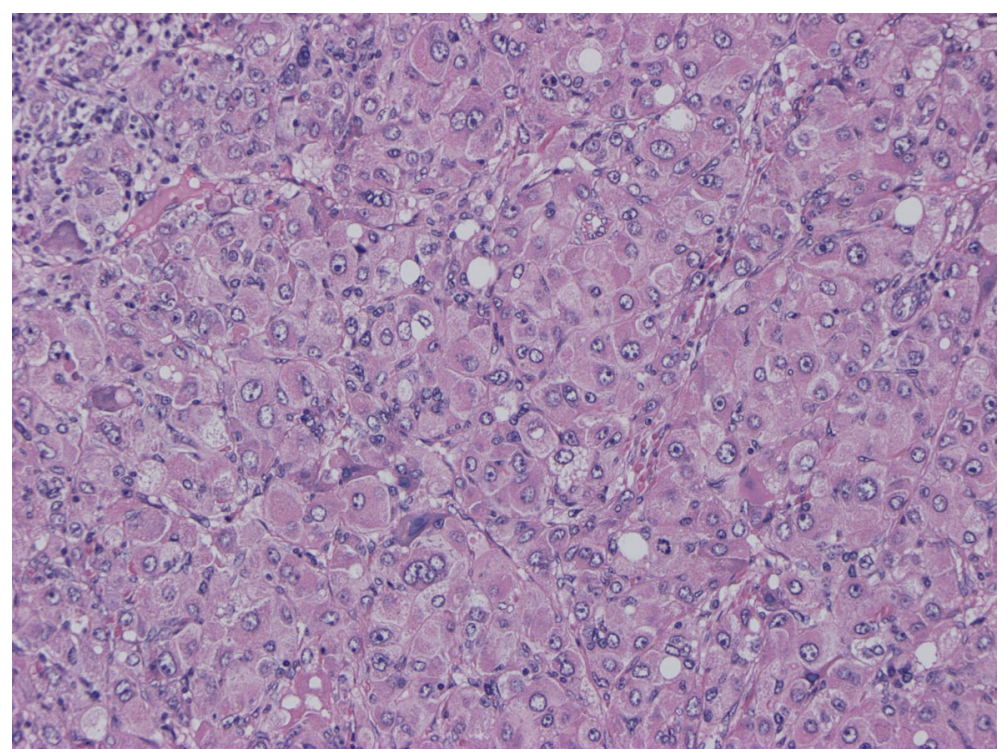




\section{References}

- Watanabe M, Matsura T, Takatori Y, Ueki K, Kobatake T, Hidaka M, Hirakawa H, Fukumoto S, Shimada Y: Five cases of ectopic liver and a case of accessory lobe of the liver. Endoscopy 1989;21:39-42.

2 Eiserth P: Beiträge zur Kenntnis der Nebenlebern. Virchows Arch A Pathol Anat Histopathol 1940;307:307-313.

3 Collan Y, Hakkiluoto A, Hästbacka J: Ectopic liver. Ann Chir Gynaecol 1978;67:27-29.

4 Liver Cancer Study Group of Japan: Follow-Up of Patients with Primary Liver Cancer. 12th Report (1992-1993). Kyoto, Shinko Publishing Co., 1996.

5 Arakawa M, Kimura Y, Sakata K, Kubo Y, Fukushima T, Okuda K: Propensity of ectopic liver to hepatocarcinogenesis: case reports and a review of the literature. Hepatology 1999;29:57-61.

-6 Gómez Sanz R, Moreno Gonzalez E, Colina Ruiz-Delgado F, Garcia-Muñoz H, Ochando Cerdan F, Gonzalez-Pinto I: Spontaneous regression of a recurrent hepatocellular carcinoma. Dig Dis Sci 1998;43:323-328.

7 Kaneko H, Takagi S, Otsuka Y, Tsuchiya M, Tamura A, Katagiri T, Maeda T, Shiba T: Laparoscopic liver resection of hepatocellular carcinoma. Am J Surg 2005;189:190-194.

$>8$ Seo UH, Lee HJ, Ryu WS, Kwak JM, Shin BK, Kim WB, Choi SY, Mok YJ: Laparoscopic resection of a hepatocellular carcinoma arising from an ectopic liver. Surg Laparosc Endosc Percutan Tech 2008;18:508-510.

-9 Gotoh K, Yamada T, Ishikawa O, Takahashi H, Eguchi H, Yano M, Ohigashi H, Tomita Y, Miyamoto Y, Imaoka S: A novel image-guided surgery of hepatocellular carcinoma by indocyanine green fluorescence imaging navigation. J Surg Oncol 2009;100:75-79.

10 Kusano M: A New Light for Minimally Invasive Surgery. Tokyo, Intermedica, 2008.

11 Harada K, Miwa M, Fukuyo T, Watanabe S, Enosawa S, Chiba T: ICG fluorescence endoscope for visualization of the placental vascular network. Minim Invasive Ther Allied Technol 2009;18:1-5. 